\title{
A simple approach to achieve organic radicals with unusual solid-state emission
}

\section{and extraordinary stability}

Xueqian Zhao ${ }^{1}$, Zheng Zhao ${ }^{1}$, Junyi Gong ${ }^{1}$, Chao $\mathrm{Ma}^{2}$, Parvej Alam ${ }^{1}$, Yanpei Wang ${ }^{3}$, Jing Guo ${ }^{3}$,

Zebin Zeng ${ }^{3}$, Zikai He ${ }^{4}$, Herman H. Y. Sung ${ }^{1}$, Ian D. Williams ${ }^{1}$, Kam Sing Wong ${ }^{2}$, Jacky W.Y.

Lam $^{1}$, Ben Zhong Tang ${ }^{1,5,6^{*}}$

${ }^{1}$ Department of Chemistry, Hong Kong Branch of Chinese National Engineering Research

Center for Tissue Restoration and Reconstruction, Institute of Molecular Functional Materials,

Division of Life Science and State Key Laboratory of Molecular Neuroscience, The Hong Kong University of Science and Technology, Clear Water Bay, Kowloon, Hong Kong 999077, China.

${ }^{2}$ Department of Physics, The Hong Kong University of Science and Technology, Clear Water Bay, Kowloon, Hong Kong 999077, China.

${ }^{3}$ State Key Laboratory of Chemo/Biosensing and Chemometrics, College of Chemistry and Chemical Engineering, Hunan University, Changsha 410082, China.

${ }^{4}$ School of Science, Harbin Institute of Technology, Shenzhen, HIT Campus of University Town, Shenzhen 518055, China.

${ }^{5}$ HKUST-Shenzhen Research Institute, Shenzhen 518057, China

${ }^{6}$ NSFC Center for Luminescence from Molecular Aggregates, SCUT-HKUST Joint Research Institute, State Key Laboratory of Luminescent Materials and Devices, South China University of Technology, Guangzhou 510640, China 


\section{Abstract}

Organic luminescent radicals are attracting much attention because of their potential applications in optoelectronics. However, most luminescent radicals prepared so far are synthesized through complicated molecular design and procedure, and in-situ generation of stable luminescent radicals is full of great challenge. All these factors have significantly limited the development of this research field. Herein, we report a simple approach to generate stable luminescent radicals in the presence of gentle UV irradiation. The newly generated radical species are capable of emitting unusual red light with a maximum fluorescence quantum efficiency of $4.6 \%$ at ambient conditions in the solid state. Additionally, the luminescent radicals show extraordinary stability with more than one-year life-span at ambient conditions. X-ray diffraction study combined with photophysical and computational analyses reveal that the unique molecular symmetry breaking in the crystalline state lead to the unusual formation and stabilization of radical ion pairs via the photo-induced electron transfer (PET) process. Such results represent the first time to achieve stable emissive radicals in the solid state at ambient conditions via in-situ PET process.

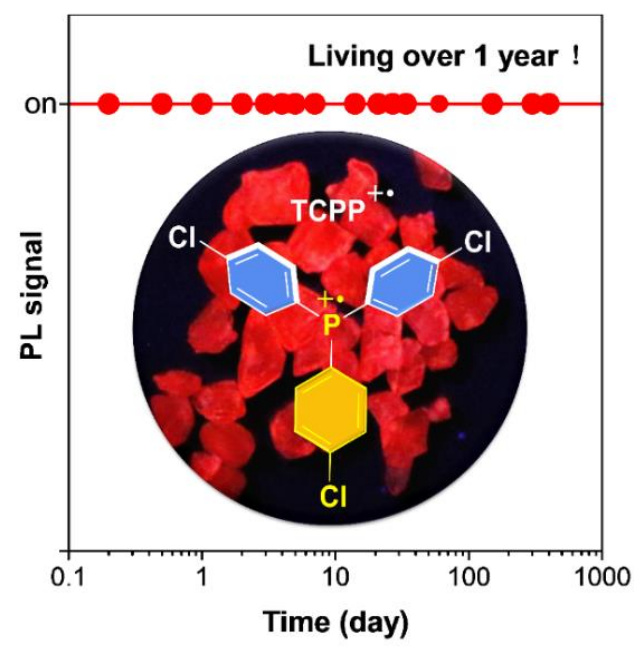




\section{Introduction}

Organic radicals with unpaired electrons have fascinated scientists for decades due to their significance in both fundamental science ${ }^{1}$ and technological applications ${ }^{2}$. Unfortunately, although they are ubiquitous in our daily life, they have been commonly correlated with pathogenic factors that cause human diseases ${ }^{3,4}$ or destructive roles to cause aging of plastic ${ }^{5}$, which leads to discrimination to organic radical species. Additionally, the radical species generally hold the characteristic of high reactivity, instability, and uncontrollability, which make the research involved in radicals difficult and challenging ${ }^{6}$. However, if we can control and stabilize these radicals and further utilize their properties, the disadvantage of radicals may become advantages and breakthroughs in both chemistry and materials may be resulted. One of the representative examples for radical utilization is the discovery and development of living radical polymerization ${ }^{7}$, which basically depends on the development of the strategy of stabilizing and controlling radical intermediate. Besides the significant application in organic catalysis or polymerization, the open-shell characteristic of organic radicals also endows them with unique magnetic and optical properties, enabling their significant applications in various advanced functional materials such as molecular magnets ${ }^{8}$, spintronics ${ }^{9}$ and optoelectronic materials ${ }^{10,11}$, etc. Unfortunately, until now, the stability issue remains a major obstacle that needs to be properly addressed for further fundamental research advancement and practical applications of organic radical species ${ }^{12}$.

Gomberg ${ }^{13}$ discovered triphenylmethyl radical as the first relative "stable" organic radical species in 1900, since then, great effort has been devoted to this area to develop more persistent radical molecules and elucidate the structure-property relationship as well as explore their 
potential applications ${ }^{14}$. With over one century of continuous exploration, various strategies, including chemical modification ${ }^{15,16}$, physical doping and even supramolecular assembling ${ }^{6,17}$, were developed to produce new radical systems and stabilize them. Representative systems include the tripenylmethyl derivative that using a sizeable steric hindrance to shield the paramagnetic center ${ }^{18}$, polycyclic aromatic hydrocarbons ${ }^{19,} 20$ that using large $\pi$-delocalization to stabilize the radicals and $\mathrm{MOF}^{21}$ that utilizes the porous and three dimensional frameworks to protect the radical species, etc. Although the variety of these generated radical molecules are still rather limited, they have begun to exhibit amazing applications in different areas. For example, Nicewicz and coworkers discovered that a neutral acridine radical could work as a superior radical phororeductant whose performance is even comparable with the lithium ${ }^{22}$. Savoie and Boudouris ${ }^{23}$ etc. fabricated a non-conjugated radical polymer by employing the commonly used TEMPO as a monomer whose conductance was as high as the value of commercial conjugated conducting polymer. These achievements suggest the great potential of organic radical species in developing advanced functional materials ${ }^{24}$.

Besides the unique reactivity and electronic properties, organic radical species also exhibit interesting luminescence property ${ }^{25,26}$ since its emission originated from a spin doublet, rather than a singlet or triplet exciton like conventional luminophores. Therefore, organic luminescent radicals are not only of fundamental research interest ${ }^{27}$ but can also be exploited in applications of fluorescence probes ${ }^{28}$ or optoelectronic devices ${ }^{29}$. In particular, the doublet emission of the radical emitter is still spin-allowed in OLEDs, which can circumvent the efficiency limitations imposed by triplet excitons to afford a theoretical $100 \%$ internal quantum yield ${ }^{30-32}$. In spite of the great prospect, however, so far, the existing radicals that are sufficiently stable to be isolated 
and stored under ambient conditions are very scarce, let alone the emissive one. Furthermore, as many of the conventional luminophores that usually show bright emission in solution state but weak or quenched emission in solid-state that has been known as aggregation-caused quenching (ACQ) effect, organic radical emitters also suffer the same issue which further increases the obstruction for application since complex chemical modification or physical manipulation is needed to overcome this ACQ effect ${ }^{11}$.

In this work, we demonstrate that the photoirradiation of tris(4-chlorophenyl)phosphine (TCPP) crystals can generate radical species, which can be stabilized in ambient conditions over one year with robust red emission. Furthermore, the emission of TCPP radical species shows aggregation-induced emission ${ }^{33}$ property, which is not emissive in solution state but brightly emissive in the solid-state without any extra chemical modification. X-ray crystallographic analysis combined with density functional theory (DFT) calculations reveals that the unique symmetry breaking of TCPP in the crystalline state result in the charge separation of the TCPP molecules ${ }^{34}$, which is the main reason that account for the generation of stable solid-state emissive radicals. Therefore, this work, for the first time, presents a simple, elegant and straightforward strategy to produce persistent and red emissive radicals in the solid-state. What's more, the TCPP radical in the crystal exhibits extraordinary stability, which can show undegraded emission even stored at ambient conditions for over one year. The photoirradiation induced generation of radical emission can be used for the anti-counterfeiting application. The current work may open up a new window of achieving stable and solid-state luminescent radical species.

\section{Results and discussion}



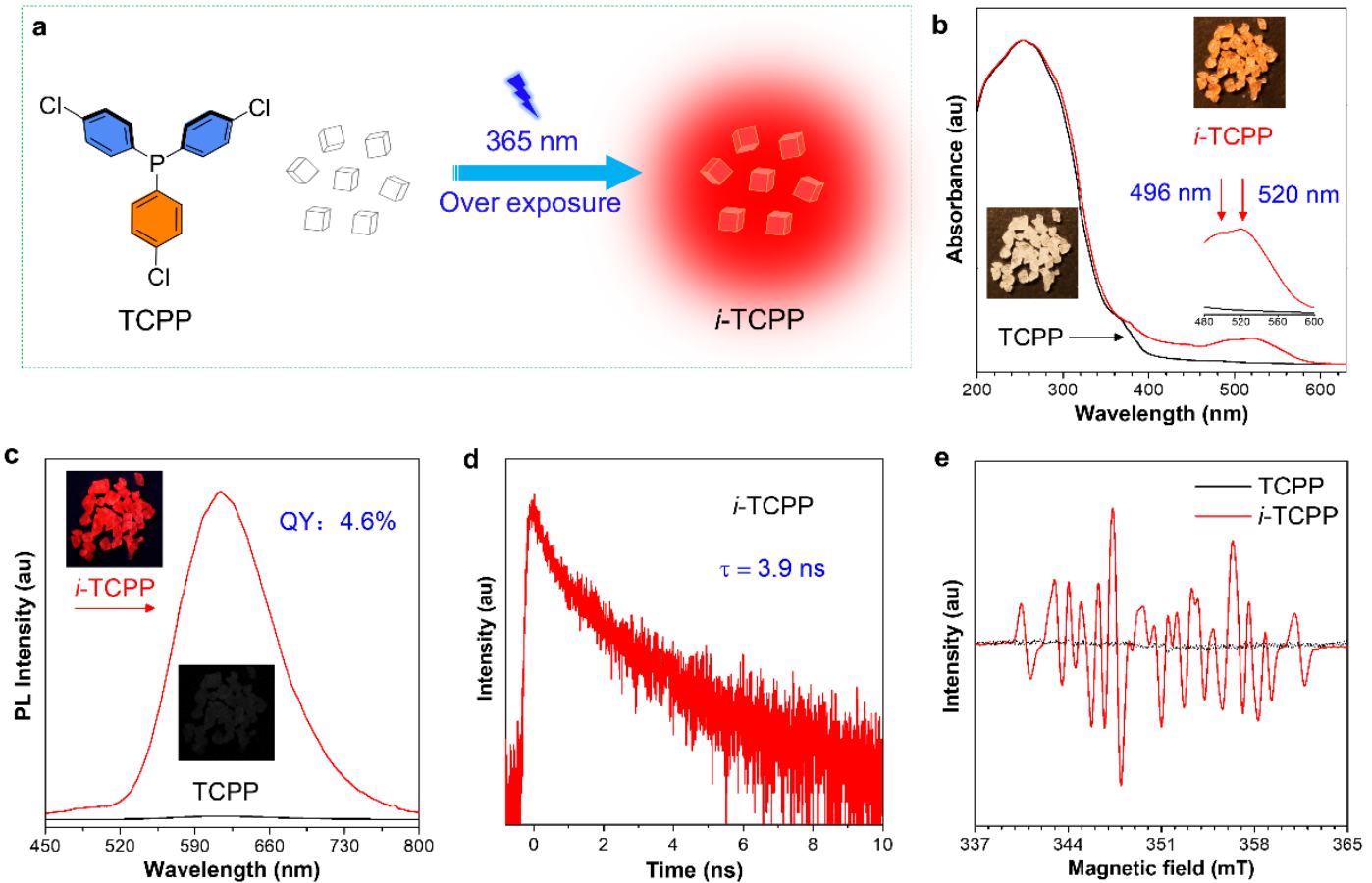

Fig. 1 Photophysical and magnetic properties of ptotochromic process a, schematic diagram of the photochromic process. b, UV-vis, $\mathbf{c}$, Photoluminescence (PL) spectra and e, electron paramagnetic resonance (EPR) spectra of TCPP crystals before (blue line) and after (red line) UV light exposure for $5 \mathrm{~min}$ at room temperature. The insets in $\mathbf{b}$, and $\mathbf{c}$, show the photographs of color and fluorescence changes. $\lambda_{\mathrm{ex}}=365 \mathrm{~nm}$. d, time-resolved PL decay curves of $i$-TCPP measured at $620 \mathrm{~nm}$ at room temperature.

TCPP sample is purchased from a commercial source that was purified by repeated chromatography and recrystallization (Fig. S1-S3). The final products were obtained as colorless cubic crystals with no emission (Fig. S4). The structure of TCPP was confirmed by NMR, HRMS spectroscopy and single-crystal structure (Fig. S5-S7). UV-vis spectroscopy measurement shows that TCPP crystal exhibits an absorption band centered at around $250 \mathrm{~nm}$, which is consistent with its colorless appearance as shown in Fig. 1b. However, upon $365 \mathrm{~nm}$ UV irradiation, the colorless crystals of the TCPP gradually become orange with two new 
absorption peaks appeared at $496 \mathrm{~nm}$ and $520 \mathrm{~nm}$, suggesting some species with a narrow bandgap was generated. Since triphenylphosphine derivatives are electron-rich species which are easy to lose one electron through oxidation to generate radical species ${ }^{35}$, oxide product or even homocoupling product to result in the narrow bandgap products, spectroscopy method thus has been implemented for the photoirradiated TCPP crystals ( $i$-TCPP) to unveil the new species generated during the photoirradiation. The results indicate that TCPP oxide was generated during the photo-irradiation according to HPLC measurement and ${ }^{31} \mathrm{P}$ NMR spectra analysis (Fig. S8). However, as TCPP oxide is also colorless rather than orange, we thus speculate that the orange color may originate from TCPP radical species because the formation of TCPP oxide will experience an intermediate of radical. Indeed, Electron paramagnetic resonance (EPR) spectroscopy measurement (Fig. 1e) indicates that $i$-TCPP crystal exhibit a strong radical signal in comparison with the radical silence property of pristine TCPP crystals. The open-shell property of the $i$-TCPP crystal was further verified by the magnetic susceptibility measurements as shown in Fig S9. Since the radicals can only be stabilized inside the crystals, those generated on the surface of the crystal thus can not be stabilized and were further oxidized by the ambient oxygen to generate TCPP oxide, which explained the TCPP oxide signal detected by HPLC. Therefore, these results indicate that the photoirradiation of TCPP crystal can induce the generation of radical species which can be stabilized inside the crystals. The photographs (Fig. S10 and S11) of $i$-TCPP crystals also show that the color change begins from the inside of the crystals and then gradually spreads to the peripheral side since those generated on the surface more difficult to be stabilized. By loading the $i$-TCPP crystals in methanol solution (Fig. S12) to evaluate the anti-degradation capability of the $i$-TCPP 
crystals, although the orange color of the radicals on the peripheral side gradually fade, while the color of the central part can still maintain for days, indicating that the dense packing of the $i$-TCPP crystal can well protect the inner radicals of the crystals from the erosion of the ambient quenching factors.

It is interesting that $i$-TCPP show bright red emission with an emission peak at $620 \mathrm{~nm}$ relative to the non-emissive characteristic of TCPP crystals without photoirradiation presented in Fig. 1c. The lifetime of $i$-TCPP crystals is $3.9 \mathrm{~ns}$ according to the transient PL spectroscopy measurement, suggesting the fluorescence characteristic of $i$-TCPP crystals (Fig. 1d). To elucidate the origin of the red luminescence, further detailed photophysical property investigation has been implemented. Because trace amount of impurity ${ }^{36}$ of may influence the luminescence property greatly, the purity of TCPP molecule is thus firstly confirmed by HPLC and elemental analysis with a deviation value lower than $0.3 \%$ relative to the theoretical value, which fulfills the requirement of criterion of American Chemical Society for the pure compound (Fig. S8a). Moreover, we also purchased the different batches of TCPP samples from different commercial sources and purified them through the same procedure, although these different batches of samples exhibit varied luminescent properties, the optical properties of the purified ones are identical and showed no luminescence, demonstrating the excellent repeatability and reliability of the results (Fig. S4). Therefore, the influence of external factors can be excluded, which also further confirmed that only the photoirradiation TCPP crystal could induce the generation of red fluorescence. Since the photoirradiation generates both radicals and TCPP oxide species and the TCPP oxide is non-emissive, the red fluorescence thus should originate from the radical species. Moreover, the fluorescence of $i$-TCPP will disappear once 
the crystal is destroyed by grinding it into amorphous powder because the radicals are sensitive to ambient conditions as shown in Fig. S13, this also further confirmed that the $i$-TCPP crystal is significant to protect the inner radicals of the crystals. It is worthy to note that although the crystal is brightly emissive, the solution of $i$-TCPP crystals in non-emissive. Therefore, the radical species is AIE-active. To the best of our knowledge, this should be the first radicalbased AIE luminophores ${ }^{37}$.

a

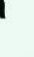

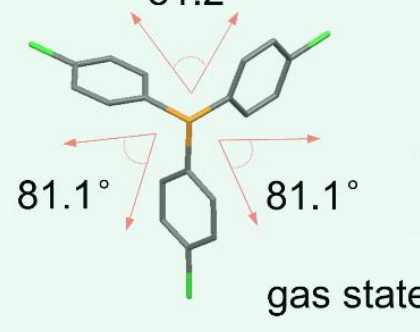

$81.2^{\circ}$

$87.6^{\circ}$

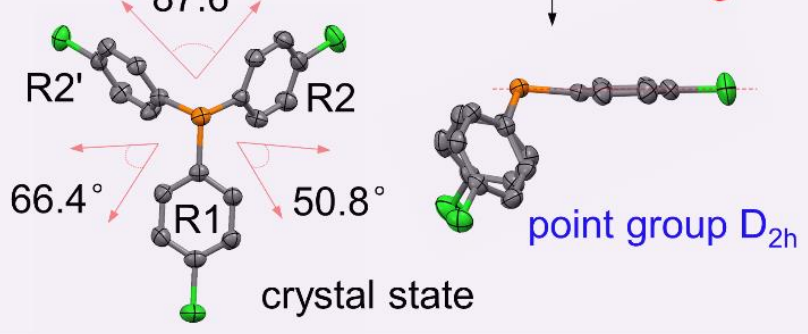

b

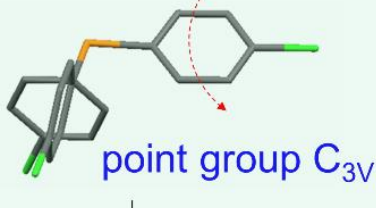

symmetry breaking

C

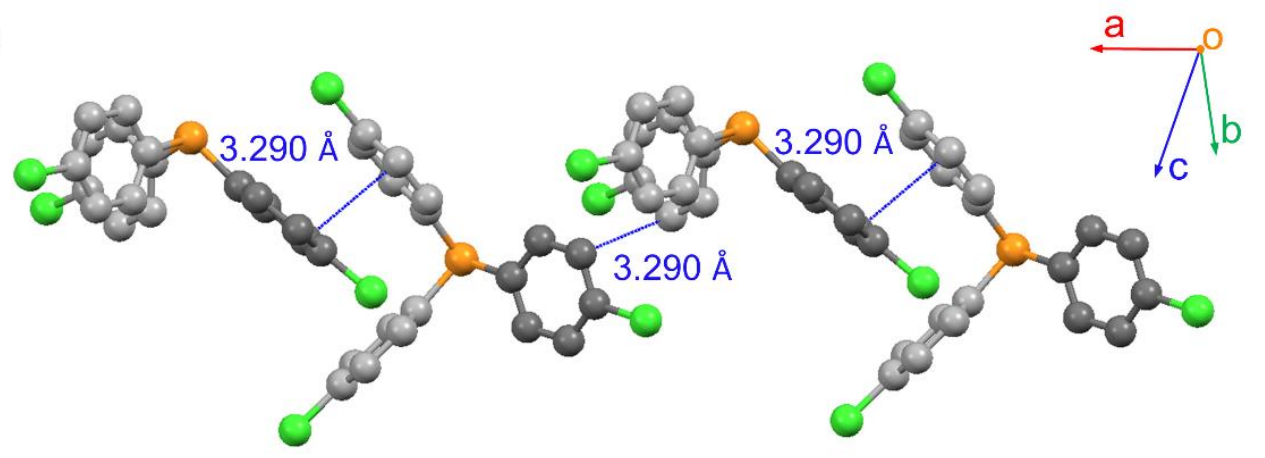

d<smiles>Clc1ccc(P(c2ccc(Cl)cc2)c2ccc(Cl)cc2)cc1</smiles> 
Fig. 2 Molecular structure analysis. a, molecular conformations of neutral TCPP obtained by DFT using BLYP/def2-SVP basis set in the (up) gas state and (down) crystal state with thermal ellipsoids set at 50\% probability. Hydrogen atoms are omitted for clarity. b, electron cloud distributions and energy levels of TCPP calculated by TD-DFT M062X/def2-TZVP, ORCA 4.1 program. $\mathbf{c}$, crystal packing diagrams of TCPP. d, proposed mechanism of photo-induced single electron transfer processes.

To further understand the optical properties and the radical generation mechanism of the TCPP molecule, we analyzed the single-crystal structure and molecular stacking pattern. Single crystal structure analysis as shown in Fig. 2a and Table S1 indicates that the molecule of TCPP adopts a $D_{2 h}$ symmetry rather than the commonly observed $C_{3 v}$ symmetry for most of its analogs like triphenylphosphine ${ }^{38}$ (Table S2). However, the optimized molecular structure of TCPP in the gas phase exhibits a $\mathrm{C}_{3 \mathrm{~V}}$ symmetry, which indicates that the crystallization-induced symmetry breaking results in the $\mathrm{D}_{2 \mathrm{~h}}$ symmetry of TCPP molecule in the crystal. Although TCPP molecule lacks typical donor-acceptor structure, density function theory (DFT) calculation based on the $\mathrm{D}_{2 \mathrm{~h}}$ symmetric structure of TCPP show obvious charge separation with the highest occupied molecular orbital (HOMO) located mainly on the phosphorous atom and phenyl ring that parallel with the plane and lowest unoccupied molecular orbital (LUMO) located mainly on the two phenyls rings vertical to the plane while TCPP in gas state with $\mathrm{C}_{3 \mathrm{~V}}$ structure shows no HOMO and LUMO separation (Fig. 2b and Fig. S14). It is worthy to note that molecules with charge separation have been reported helpful for the generation of radicals through the photo-induced charge transfer process ${ }^{39,40}$, although the generated radicals mostly are transient and sensitive, which can't exist at ambient conditions stably ${ }^{41}$. However, as a 
contrast, the radicals generated within TCPP crystals show excellent stability at ambient conditions, which suggested that the superior stability of $i$-TCPP radicals may be correlated with its crystal packing. Further crystal arrangement analysis indicates that the molecules of TCPP adopt an alternant intermolecular donor-acceptor arrangement, which should also be beneficial for the exciton separation and stabilization through the electron hopping mechanism (Fig. 2c). In consideration of the superior stability of the radicals within $i$-TCPP crystals and the unique symmetry breaking ${ }^{42}$ caused molecular arrangement as well as the fact that single molecule of TCPP can stabilize the radicals, we propose that an intermolecular radical pair model through the PET process is responsible for the radical generation and stabilization of $i$ TCPP crystal (Fig. 2d). 


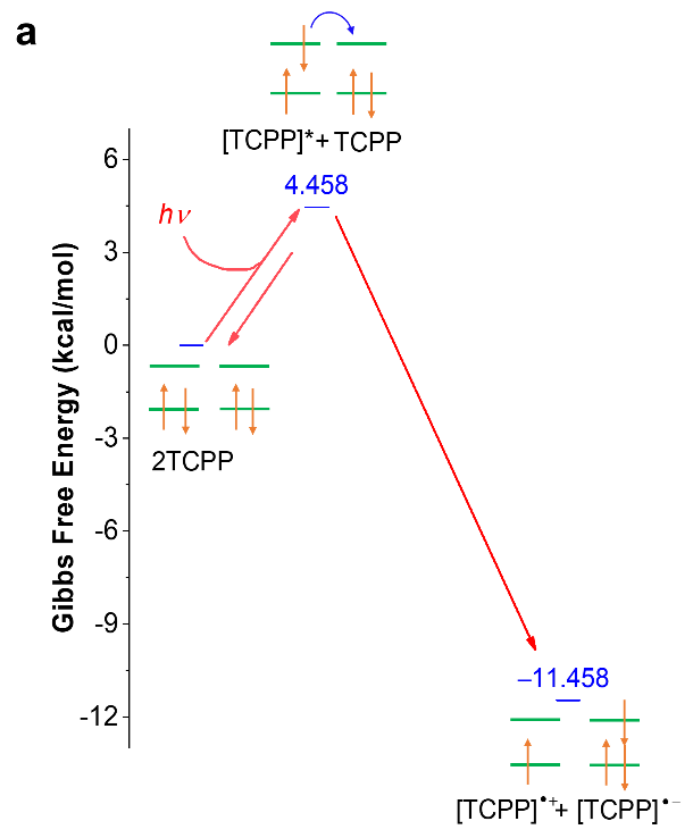

b

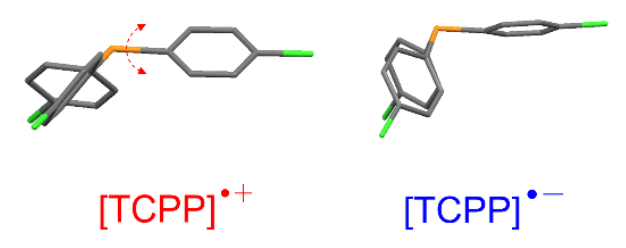

c
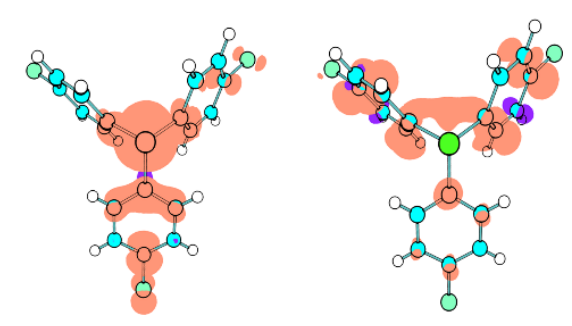

$[\mathrm{TCPP}]^{\bullet+}$
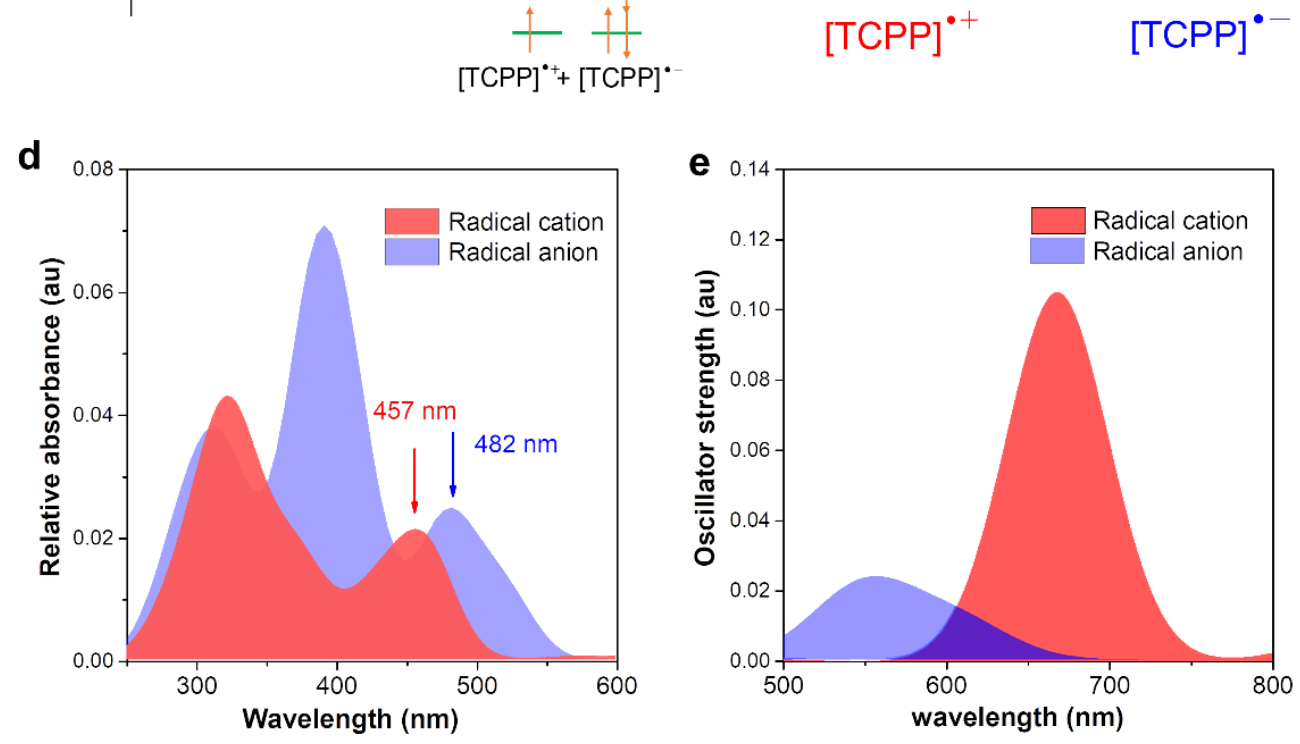

Fig. 3 DFT calculation analysis. a, a schematic diagram showing the free energy profile of the process. $\mathbf{b}$, molecular structures of TCPP radical cation and radical anion obtained by DFT using the BLYP/def2-SVP basis set. $\mathbf{c}$, the spin density distribution of TCPP radical anion and cation. d-e, absorption and emission spectra of TCPP radical anion and cation based on the quantum calculation results.

To confirm the rationality of our hypothesis, the theoretical calculation has been implemented based on the high precision double-hybrid functional PWPB95 with a def2-TZVP basis $\operatorname{set}^{43}$. We first calculated the Gibbs free energy of dimer of TCPP molecules and the radical pairs of 
TCPP molecules in the ground state as well as that of the dimer in the excited state (Fig. 3a). The results indicate that the radical pairs show much lower Gibbs free energy than the dimer in both ground state and excited state, suggesting that the formation of radical pairs are thermodynamically favorable. Interestingly, the optimized molecular configuration of radical anion of TCPP is almost identical to that of TCPP in the crystal, with both of which exhibit a $\mathrm{D}_{2 \mathrm{~h}}$ symmetry (Fig. 3b and Table S3). Therefore, the molecular configuration of TCPP within the crystal benefits the formation of the radical anion. The spin distribution of the TCPP radical pair in the crystal structure was also calculated (Fig. 3c). For the TCPP radical cation, the unpaired electron is mainly distributed on the phosphor atom and the benzene ring, which is parallel with the principal plane. While for the TCPP radical anion, the unpaired electron is distributed on the other benzene rings that vertical to the plane. Such distributions suggest that the radical species within the TCPP crystals is not homogenous but multiple, which is consistent with the complexly split EPR signals with an asymmetric hyperfine pattern. The complex EPR signal may arise from the superposition of the radical pairs with different $g_{\mathrm{e}}$ values ${ }^{44}$. The absorption and emission spectra of TCPP radical pairs are also simulated by TD-DFT and plotted in Fig. 3d and Fig. 3e. Interestingly, the superimposed absorption spectra of TCPP radical cation and anion show two absorption peaks at $457 \mathrm{~nm}$ and $482 \mathrm{~nm}$, which matched well with the experimental data with two peaks at $496 \mathrm{~nm}$ and $520 \mathrm{~nm}$ and suggested the reliability of the calculation results. Further, in-depth analysis indicates that the two absorption peaks at $457 \mathrm{~nm}$ and $482 \mathrm{~nm}$ are arisen from HOMO-9 $\rightarrow$ SOMO of radical cation and SOMO $\rightarrow$ LUMO+7 of the radical anion (Fig. 3d), respectively. The detailed energy level diagrams and wavefunctions of the frontier molecular orbitals of TCPP radical anion and cation 
are shown in Fig. S15. The emission spectra of TCPP radical cation and anion are also calculated with two emission bands centered at around $667 \mathrm{~nm}$ and $549 \mathrm{~nm}$, respectively, with oscillator strengths $(f)$ of up to 0.105 (SOMO $\rightarrow \mathrm{HOMO}-4)$ and 0.021 (LUMO+7 $\rightarrow \mathrm{SOMO}$ ), respectively. (Table S4 and Fig. S16). Therefore, the red PL of $i$-TCPP crystal mainly originated from its radical cations.
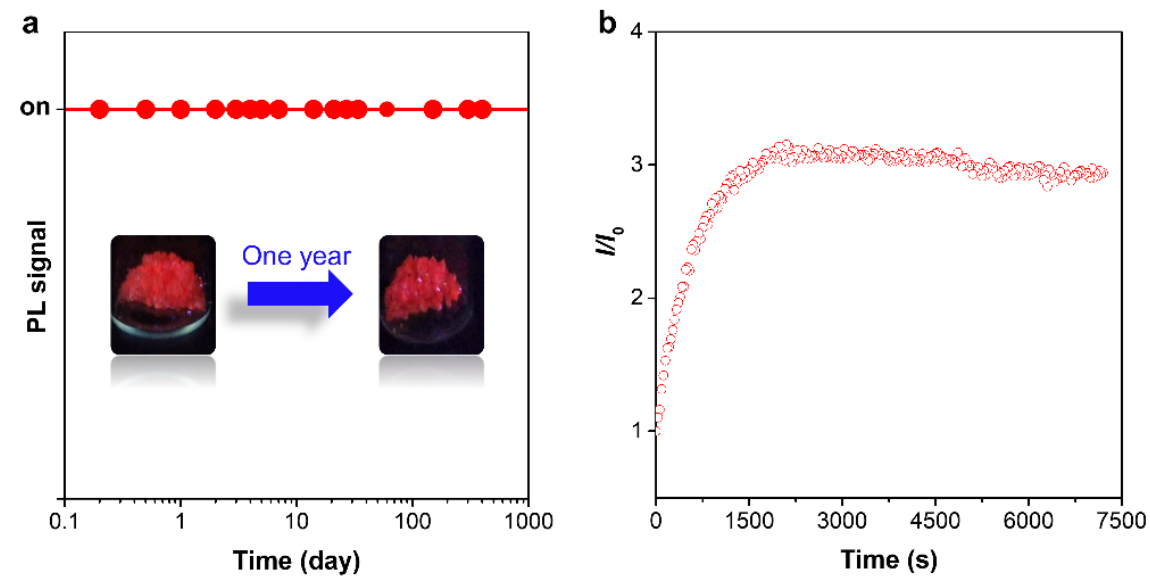

Fig. 4 The stability of TCPP radical species. a, PL signal at $620 \mathrm{~nm}$ under ambient condition, where "1" represents the exist of fluorescent signal. b, PL intensity of $i$-TCPP crystals under $400 \mathrm{~nm}$ laser radiation (power: $3 \mu \mathrm{J} / \mathrm{cm}^{2}$ ), where $I_{0}=\mathrm{PL}$ intensity of pristine $i$-TCPP. The inset shows the photographs of fluorescence changes.

The stability issue is still a bottleneck encountered by most reported radical species produced by both chemical and physical methods. However, $i$-TCPP crystal seems to exhibit extraordinary stability at ambient conditions. Besides, its bright red fluorescence in the solidstate not only has the potential for the application of advanced optical crystalline materials but also enables it possible to evaluate its stability through a visualization method by measuring the PL change with time. As shown in Fig. 4a, just locating the $i$-TCPP crystal in ambient conditions without any protection, the fluorescence signal of the crystals detected by both PL 
machine and naked eyes in different interval times showed no noticeable intensity degradation even after one year's storage. More interestingly, the PL intensity of $i$-TCPP crystal can be even further enhanced by three folds when exposed to a pulsed laser (Fig. 4b), suggesting that the number of generated radicals can be increased by strengthening the power of the irradiation source, which is also distinguished from conventional radical materials that are easy to be bleached by a strong laser pulse. By continuously laser irradiation for up to $7000 \mathrm{~s}$, the PL intensity loss is within $5 \%$.

The photoirradiation-induced generation of radical species along with color and emission change makes it possible to utilize the TCPP for anti-counterfeiting applications (Fig. S18). First, a filter paper was immersed in a dichloromethane solution of TCPP. After half-drying at ambient conditions, a seal was used to pattern a Chinese character (tang) on the paper. The word is invisible in the daylight but appears in red upon UV irradiation, which can be erased by further green light irradiation.

\section{Conclusions}

In summary, the unique molecular conformation and photo-redox property of TCPP crystal make it responsive to UV irradiation. The tightly packed structure prevents the interference from water and oxygen to significantly improve the stability of the newly formed radical ion pairs. Noteworthily, this is the first time that triarylphosphorus radicals are discovered to emit light. Results from theoretical calculations are consistent with the experimental data that TCPP radical cation is responsible for the red emission. The present results not only draw a clear mechanistic picture for the in-situ generation of stable radical ion pairs but also change the cognition of the scientific community toward classical photophysical phenomenon. Moreover, 
it provides a reliable strategy and opportunity to develop new luminescent radicals with potential applications in the fields of optical memory, multi-color displays, multiplexed optical chemical sensing, anti-counterfeiting, etc.

\section{Conflicts of Interest}

The authors declare no competing financial interest.

\section{Authors' Contributions}

Ben Zhong Tang supervised the project and conceived the project design. Xueqian Zhao, Zheng Zhao and Junyi Gong contributed equally to this work. Chao Ma, Parvej Alam, Yanpei Wang, Jing Guo, Shuai Zhang, Herman H. Y. Sung, Zikai He, Zebin Zeng, Ian D. Williams, Kam Sing Wong gave helpful discussion and some technical support to this work. Xueqian Zhao wrote the manuscript. Zheng Zhao and Jacky W.Y. Lam helped to revised the manuscript. Junyi Gong was reponsible for DFT calculations. All authors have given approval to the final version of the manuscript.

\section{Acknowledgments}

This project was financially supported by the National Natural Science Foundation of China (21788102), the Natural Science Foundation of Guangdong Province (2019B121205002 and 2019B030301003), the Research Grants Council of Hong Kong (16305618, 16305518, C6009-17G and AoE/P-02/12), the National Key Research and Development Program(2018YFE0190200), the Innovation and Technology Commission (ITCCNERC14SC01), and the Science and Technology Plan of Shenzhen (JCYJ20180306174910791, and JCYJ20170818113530705 and JCYJ20170818113538482, and JCYJ20160229205601482 ). We thank Lili Du, PHILLIPS, David Lee in the University of 
Hong Kong (HKU), Zhijiao Tang in Shenzhen University, Congwu Ge, Xike Gao in Shanghai Institute of Organic Chemistry (SIOC) Chinese Academy of Sciences (CAS), Qiang Wei in Ningbo Institute of Industrial Technology (CNITECH), Chinese Academy of Sciences (CAS), Ruoyao Zhang in the Hong Kong University of Science and Technology for the experimental measurements.

\section{References}

1. Yan M., Lo J. C., Edwards J. T. \& Baran P. S. Radicals: Reactive intermediates with translational potential. J. Am. Chem. Soc. 138, 12692-12714 (2016).

2. Ratera I. \& Veciana J. Playing with organic radicals as building blocks for functional molecular materials. Chem. Soc. Rev. 41, 303-349 (2012).

3. Halliwell, B., \& Gutteridge, J. M. The importance of free radicals and catalytic metal ions in human diseases. Molecular aspects of medicine, 8, 89-193 (1985).

4. Lunec, J. Free radicals: their involvement in disease processes. Ann. Clin. Biochem. 27, 173-182 (1990).

5. Mesarovic, S. D. Dynamic strain aging and plastic instabilities. J. Mech. Phys. Solids, 43, 671-700 (1995).

6. Sakamaki D., Ghosh S. \& Seki S. Dynamic covalent bonds: approaches from stable radical species. Mater. Chem. Front. 3, 2270-2282 (2019). 
7. Wang J. S. \& Matyjaszewski K. Controlled/“living” radical polymerization. Atom transfer radical polymerization in the presence of transition-metal complexes. J. Am. Chem. Soc. 117, 5614-5615 (1995)

.8. Gallagher N. M., Bauer J. J., Pink M., Rajca S. \& Rajca A. High-spin organic diradical with robust stability. J. Am. Chem. Soc. 138, 9377-9380 (2016).

9. Rugg B. K., et al. Photodriven quantum teleportation of an electron spin state in a covalent donor-acceptor-radical system. Nat. Chem. 11, 981-986 (2019).

10. Zhang Y., et al. Temperature tunable self-doping in stable diradicaloid thin-film devices. Adv. Mater. 27, 7412-7419 (2015).

11. Kimura S., et al. Magnetoluminescence in a photostable, brightly luminescent organic radical in a rigid environment. Angew. Chem. Int. Ed. 57, 12711-12715 (2018).

12. Power P. P. Persistent and stable radicals of the heavier main group elements and related species. Chem. Rev. 103, 789-810 (2003).

13. Gomberg M. An instance of trivalent carbon: Triphenylmethyl. J. Am. Chem. Soc. 22, 757-771 (1900).

14. Ballester, M., Riera-Figueras, J., Castaner, J., Badfa, C., \& Monso, J. M. Inert carbon free radicals. I. Perchlorodiphenylmethyl and perchlorotriphenylmethyl radical series. $J$. Am. Chem. Soc., 93, 2215-2225 (1971).

15. Back O., Donnadieu B., Parameswaran P., Frenking G. \& Bertrand G. Isolation of crystalline carbene-stabilized $\mathrm{p}(2)$-radical cations and $\mathrm{p}(2)$-dications. Nat. Chem. 2, 369-373 (2010). 
16. Wang Y., Rajca S. \& Rajca A. Pegylated, water-soluble, stable aminyl radical. J. Org. Chem. 82, 7512-7518 (2017).

17. Tang B., Zhao J., Xu J.-F. \& Zhang X. Tuning the stability of organic radicals: from covalent approaches to non-covalent approaches. Chem. Sci. 11, 1192-1204 (2020).

18. Pan X., Chen X., Li T., Li Y. \& Wang X. Isolation and X-ray crystal structures of triarylphosphine radical cations. J. Am. Chem. Soc. 135, 3414-3417 (2013).

19. Yang K., et al. Stable organic diradicals based on fused quinoidal oligothiophene imides with high electrical conductivity. J. Am. Chem. Soc. 142, 4329-4340 (2020).

20. Sun Z., Huang K. W. \& Wu J. Soluble and stable heptazethrenebis(dicarboximide) with a singlet open-shell ground state. J. Am. Chem. Soc. 133, 11896-11899 (2011).

21. Lu B., et al. Stable radical anions generated from a porous perylenediimide metal-organic framework for boosting near-infrared photothermal conversion. Nat. Commun. 10, 767 (2019).

22. MacKenzie I. A., et al. Discovery and characterization of an acridine radical photoreductant. Nature 580, 76-80 (2020).

23. Yongho J. V. A., Seung H. S., Brett M. S. \& Bryan W. B. A nonconjugated radical polymer glass with high electrical conductivity. Science 359, 1391-1395 (2018).

24. Janoschka T., et al. An aqueous, polymer-based redox-flow battery using non-corrosive, safe, and low-cost materials. Nature 527, 78-81 (2015).

25. Imran M., Wehrmann C. M. \& Chen M. S. Open-shell effects on optoelectronic properties: antiambipolar charge transport and anti-kasha doublet emission from a nsubstituted bisphenalenyl. J. Am. Chem. Soc. 142, 38-43 (2020). 
26. Beldjoudi Y., et al. Multifunctional dithiadiazolyl radicals: fluorescence, electroluminescence, and photoconducting behavior in pyren-1'-yl-dithiadiazolyl. J. Am. Chem. Soc. 140, 6260-6270 (2018).

27. Teki Y. Excited-state dynamics of non-luminescent and luminescent pi-radicals. Chem. Eur.J. 26, 980-996 (2020).

28. Ishii K., Kubo K., Sakurada T., Komori K. \& Sakai Y. Phthalocyanine-based fluorescence probes for detecting ascorbic acid: Phthalocyaninatosilicon covalently linked to tempo radicals. Chem. Commun., 47, $4932-4934$ (2011).

29. Reig M., et al. Stable all-organic radicals with ambipolar charge transport. Chem. Eur. J. 22, 18551-18558 (2016).

30. Guo H., et al. High stability and luminescence efficiency in donor-acceptor neutral radicals not following the aufbau principle. Nat. Mater. 18, 977-984 (2019).

31. Ai X., et al. Efficient radical-based light-emitting diodes with doublet emission. Nature 563, 536-540 (2018).

32. Peng Q., Obolda A., Zhang M. \& Li F. Organic light-emitting diodes using a neutral pi radical as emitter: The emission from a doublet. Angew. Chem. Int. Ed., 54, 7091-7095 (2015).

33. Mei J., Leung N. L., Kwok R. T., Lam J. W. \& Tang B. Z. Aggregation-induced emission: Together we shine, united we soar! Chem. Rev. 115, 11718-11940 (2015).

34. Bin Shawkataly O., Singh, J., Fun, H. K. \& Sivakumar, K. Tris (4-chlorophenyl) phosphine and tris (4-fluorophenyl) phosphine. Acta Crystallographica C. 52, 2243$2245(1996)$ 
35. Yasui S., Kobayashi S. \& Mishima M. Comprehensive investigation on the reactivity of triarylphosphine radical cations by laser flash photolysis time-resolved uv-vis spectroscopy. J. Phys. Org. Chem. 29, 443-451 (2016).

36. Chen, C. J., et al. Impurity Conundrum of Organic Room Temperature Afterglow. ChemRxiv. Preprint at https://doi.org/10.26434/chemrxiv.9895724.v1 (2019).

37. Xu K., et al. Photo-induced free radical production in a tetraphenylethylene ligand-based metal-organic framework. Chem. Commun. 54, 12942-12945 (2018).

38. Triphenylphosphine: A redetermination. Acta Crystallographica C. 47, 345-347 (1991).

39. Sindt A. J., et al. UV-irradiation of self-assembled triphenylamines affords persistent and regenerable radicals. Chem. Sci. 10, 2670-2677 (2019).

40. Lambert C., et al. A photoinduced mixed-valence state in an organic bis-triarylamine mixed-valence compound with an iridium-metal-bridge. Chem. Commun., 50, 1135011353 (2014).

41. Dereka B., Koch M. \& Vauthey E. Looking at photoinduced charge transfer processes in the IR: Answers to several long-standing questions. Acc Chem Res., 50, 426-434 (2017).

42. Lee C., Choi C. H. \& Joo T. A solvent-solute cooperative mechanism for symmetrybreaking charge transfer. Phys. Chem. Chem. Phys. 22, 1115-1121 (2020).

43. Neese F. Software update: The orca program system, version 4.0. WIREs Comput Mol. Sci. 8, 1327 (2018).

44. Gerson. F. \& Huber. W. Electron spin resonance spectroscopy of organic radicals. John Wiley \& Sons, (2003). 
